\title{
Predictors of Locoregional Recurrence After Failure to Achieve Pathologic Complete Response to Neoadjuvant Chemotherapy in Triple-Negative Breast Cancer
}

\author{
Prashant Gabani, MD, ; Emily Merfeld, MD, ; Amar J. Srivastava, MDa; Ashley A. Weiner, MD, PhD; \\ Laura L. Ochoa, RN, PhDa; Dan Mullen, DDSa; Maria A. Thomas, MD, PhDa; Julie A. Margenthaler, MD; Amy E. Cyr, MDc; \\ Lindsay L. Peterson, MDd; Michael J. Naughton, MDd; Cynthia Ma, MDd; and Imran Zoberi, MDa
}

\begin{abstract}
Background: This study evaluated factors predictive of locoregional recurrence (LRR) in women with triple-negative breast cancer (TNBC) treated with neoadjuvant chemotherapy who do not experience pathologic complete response ( $\mathrm{PCR})$. Methods: This is a singleinstitution retrospective review of women with TNBC treated with neoadjuvant chemotherapy, surgery, and radiation therapy in 2000 through 2013. LRR was estimated between patients with and without pCR using the Kaplan-Meier method. Patient-, tumor-, and treatmentspecific factors in patients without $\mathrm{PCR}$ were analyzed using the Cox proportional hazards method to evaluate factors predictive of LRR. Log-rank statistics were then used to compare LRR among these risk factors. Results: A total of 153 patients with a median follow-up of 48.6 months were included. The 4-year overall survival and LRR were $70 \%$ and $15 \%$, respectively, and the 4 -year LRR in patients with $\mathrm{PCR}$ was $0 \%$ versus $22.0 \%$ in those without $(P<.001)$. In patients without $\mathrm{pCR}$, lymphovascular space invasion (LVSI; hazard ratio, 3.92; $95 \% \mathrm{Cl}$, 1.64-9.38; $P=.002$ ) and extranodal extension (ENE; hazard ratio, $3.32 ; 95 \% \mathrm{Cl}, 1.35-8.15 ; P=.009)$ were significant predictors of $L R R$ in multivariable analysis. In these patients, the 4 -year LRR with LVSI was $39.8 \%$ versus $15.0 \%$ without $(P<.001)$. Similarly, the 4 -year LRR was $48.1 \%$ with ENE versus $16.1 \%$ without $(P=.002)$. In patients without $\mathrm{pCR}$, the presence of both LVSI and ENE were associated with an even further increased risk of LRR compared with patients with either LVSI or ENE alone and those with neither LVSI nor ENE in the residual tumor $(P<.001)$. Conclusions: In patients without $P C R$, the presence of LVSI and ENE increases the risk of LRR in TNBC. The risk of LRR is compounded when both LVSI and ENE are present in the same patient. Future clinical trials are warranted to lower the risk of LRR in these high-risk patients.
\end{abstract}

J Natl Compr Canc Netw 2019;17(4):348-356 doi: 10.6004/jnccn.2018.7103

aDepartment of Radiation Oncology, Washington University School of Medicine, Saint Louis, Missouri; ${ }^{b}$ Department of Radiation Oncology, University of North Carolina, Chapel Hill, North Carolina; and 'Department of Surgery, and ${ }^{\mathrm{d} D e p a r t m e n t}$ of Medicine, Washington University School of Medicine, Saint Louis, Missouri.

*These authors contributed equally.

\section{Background}

Breast cancer is a heterogeneous group of diseases and encompasses a variety of molecular and morphologic subtypes. Triple-negative breast cancer (TNBC) represents approximately $15 \%$ to $20 \%$ of all breast cancers. ${ }^{1}$ Compared with hormone receptor-positive breast cancer, TNBC follows an aggressive clinical course with a shorter disease-free interval and overall survival. ${ }^{1,2}$ In addition, a higher rate of locoregional recurrence (LRR) has been reported for TNBC. ${ }^{3-5}$

Patients with locally advanced TNBC typically receive chemotherapy, surgery, and radiation therapy (RT). More recently, the concept of neoadjuvant chemotherapy (NAC) followed by surgery and RT has been introduced in women with locally advanced or palpable TNBC, ${ }^{6}$ because TNBC has been shown to have greater chemosensitivity than other breast cancer subtypes. ${ }^{1,5}$ Patients who achieve a pathologic complete response (pCR) after NAC have been reported to have significantly improved survival outcomes and locoregional control compared with those with residual disease. ${ }^{7,8}$ However, in those receiving NAC, the predictive value of other pathologic or treatment-related factors for local control is unclear because data are lacking.

Patients with residual disease after NAC have a 2- to 3-fold higher relative risk of developing $\mathrm{LRR}^{8}$; however, most remain free of LRR. ${ }^{8}$ For that reason, identifying patients with residual disease after NAC who are at high risk for LRR is clinically meaningful because it allows close monitoring for recurrence and early incorporation of salvage treatments..$^{9,10}$ Therefore, this study aimed to evaluate factors predictive of LRR in patients with TNBC who have residual disease and therefore do not experience pCR after NAC.

See JNCCN.org for supplemental online content. 


\section{Methods}

An Institutional Review Board-approved institutional database was used as a source for this analysis. All consecutive patients with TNBC who were treated with NAC, surgery, and RT in 2000 through 2013 were identified. TNBC was defined by the lack of estrogen and progesterone receptor expression and lack of HER2 expression or gene amplification. Patients with metastatic disease at diagnosis and those who developed progressive disease during treatment were excluded.

Patient-, tumor-, and treatment-specific factors were analyzed, including age, race, smoking history; tumor stage, histology, and grade; and type of NAC used. Before NAC, all patients were staged clinically by physical examination along with incorporation of diagnostic imaging. After NAC, all patients were staged surgically. Downstaging was defined as a decrease in $\mathrm{T}$ stage, $\mathrm{N}$ stage, or both after NAC (eg, clinical T3 disease treated with NAC and subsequently staged as $\leq \mathrm{T} 2$ based on final pathology). pCR was defined as no evidence of invasive disease in the breast or regional lymph nodes after NAC. LRR was defined as reappearance of cancer in the ipsilateral breast, chest wall, or regional lymph nodes. The presence or absence of lymphovascular space invasion (LVSI), extranodal extension (ENE), or pathologically positive lymph nodes was determined during surgery. All patients in this database were treated with whole-breast or chest wall RT with or without regional nodal irradiation (RNI). RNI was delivered to $81 \%$ of patients. RNI at our institution includes treating levels I, II, and III of the axilla, interpectoral, supraclavicular, and internal mammary lymph nodes. It is our institutional practice to use 3D tangents for whole-breast RT and (infrequently) the deep inspiration breath hold technique. Before 2007, RNI was delivered using 3D tangents with or without internal mammary field, with supraclavicular field. This practice shifted to using intensity-modulated RT after 2007.

Time intervals for the analysis were calculated from date of diagnosis. Overall survival and LRR were estimated using the Kaplan-Meier method. Rates of LRR for patients who achieved pCR and those who did not were compared using log-rank statistics. Univariable and multivariable Cox proportional hazards methods were used to evaluate factors predictive of LRR in patients who did not experience pCR (ie, those with residual disease) after NAC. Stepwise regression, a modification of the forward selection method with selection criterion $P<.05$ based on Cox regression models, was applied to obtain the final multivariable model. In patients who did not experience pCR, the Kaplan-Meier method was used to estimate whether those with multiple risk factors had an increased risk of LRR compared with those with a single risk factor. Statistical analysis was performed using SPSS
Statistics, version 25 (IBM Corporation), and $P<.05$ was considered statistically significant.

\section{Results}

A total of 153 patients were included in the analysis; baseline characteristics are shown in Table 1. Median age at diagnosis was 49.5 years (range, 28.8-77.3 years). The most common NAC regimen was doxorubicin/ cyclophosphamide/paclitaxel (AC-T; $n=62 ; 40.5 \%$ ). Patients receiving epirubicin/paclitaxel (ET) were part of an institutional phase II trial. ${ }^{11}$ Adjuvant chemotherapy was also administered in 46 patients $(30.1 \%)$. A total of 73 patients $(47.7 \%)$ underwent breast-conserving surgery, and $118(77.1 \%)$ underwent axillary lymph node dissection. The rate of pCR was $29.4 \%(n=45)$. Most patients $(81.0 \%)$ received comprehensive $\mathrm{RT}$, including treatment of the whole breast or chest wall along with regional nodes. The median RT dose was $50.4 \mathrm{~Gy}$, and a sequential boost was delivered in 101 patients $(66.0 \%)$.

Median follow-up was 48.6 months (range, 6.1-167.6 months). At the time of analysis, 49 patients had died. The 4-year overall survival was $70 \%$ for the whole cohort (Figure 1A), and the 4-year progression-free survival was $67.9 \%$ (see supplemental eFigure 1, available with this article at JNCCN.org). A total of 21 patients developed LRR, including 5 in-breast recurrences, 11 nodal recurrences, and 11 chest wall recurrences. Of the 11 nodal recurrences, failure occurred in the axilla in 8 patients and in the axilla and other nodal regions in 3 patients. There were no isolated internal mammary failures. The 4-year locoregional control for the whole cohort was $85 \%$ (Figure 1B). Among the 45 patients who experienced pCR, none had LRRs. Conversely, in the 108 patients without pCR, 21 (19.4\%) had LRRs. The 4-year rate of locoregional control in patients who achieved pCR was $100 \%$ versus $78 \%$ in those who did not achieve pCR after NAC (Figure 2A) $(P<.001)$.

To identify factors predictive of LRR, univariable and multivariable analyses were performed on patients who had residual disease after NAC (summarized in Table 2). On univariable analysis, LVSI and ENE were associated with LRR. On multivariable analysis, LVSI (hazard ratio, 3.92; 95\% CI, 1.64-9.38) and ENE (hazard ratio, 3.32; 95\% CI, 1.35-8.15) both remained as independent factors predictive of LRR. The rate of locoregional control at 4 years was $61.2 \%$ for patients with LVSI versus $85.0 \%$ in patients without LVSI after NAC (Figure 2B) $(P<.001)$ The rate of locoregional control at 4 years was $51.9 \%$ in patients with ENE versus $83.9 \%$ in patients without ENE after NAC (Figure 2C) $(P=.002)$.

Only 10 patients had both LVSI and ENE present in their residual disease. Of these, 5 (50\%) developed LRR. There were 35 patients who had either LVSI or ENE, but not both. Of these, 10 (28.6\%) developed LRR. There were 


\section{Table 1. Baseline Patient Characteristics}

\section{Characteristic}

n (\%)

All patients

$153(100)$

Age at diagnosis, $y$

\begin{tabular}{ll}
\hline$<50$ & $78(51.0)$ \\
$\geq 50$ & $75(49.0)$
\end{tabular}

Race

\begin{tabular}{ll}
\hline White & $86(56.2)$ \\
\hline Black & $67(43.8)$
\end{tabular}

Menopausal status

Premenopausal

$78(51.0)$

Postmenopausal

$75(49.0)$

Clinical T stage

\begin{tabular}{ll}
\hline $\mathrm{T} 1$ & $12(7.8)$ \\
\hline $\mathrm{T} 2$ & $66(43.1)$ \\
$\mathrm{T} 3$ & $44(28.8)$ \\
\hline $\mathrm{T} 4$ & $31(20.3)$
\end{tabular}

T4

$31(20.3)$

Clinical $\mathrm{N}$ stage

\begin{tabular}{lr}
\hline N0-1 & $120(78.4)$ \\
\hline N2-3 & $33(21.6)$
\end{tabular}

Multifocal

\begin{tabular}{lr} 
No & $133(86.9)$ \\
\hline Yes & $20(13.1)$
\end{tabular}

Histology

\begin{tabular}{lr}
\hline Ductal & $135(88.2)$ \\
\hline Other & $18(11.8)$
\end{tabular}

Grade

I or II 25 (16.3)

III

$128(83.7)$

Pathologic complete response

\begin{tabular}{lr} 
No & $108(70.6)$ \\
\hline Yes & $45(29.4)$
\end{tabular}

Pathologic T stage

\begin{tabular}{ll}
\hline T0 & $57(37.3)$ \\
\hline T1 & $44(28.8)$ \\
T2 & $28(18.3)$ \\
\hline T3 & $13(8.5)$ \\
\hline T4 & $11(7.2)$
\end{tabular}

Pathologic N stage

\begin{tabular}{ll}
\hline $\mathrm{N} 0$ & $93(60.8)$ \\
\hline $\mathrm{N} 1$ & $32(20.9)$ \\
\hline $\mathrm{N} 2$ & $15(9.8)$ \\
\hline $\mathrm{N} 3$ & $13(8.5)$
\end{tabular}

LVSI

\begin{tabular}{lr}
\hline Absent & $119(77.8)$ \\
\hline Present & $34(22.2)$ \\
\hline
\end{tabular}

(continued)

\section{Table 1. Baseline Patient Characteristics (cont.)}

Characteristic

n (\%)

Margins

\begin{tabular}{lc}
\hline Negative & $143(93.5)$ \\
\hline Positive & $10(6.5)$
\end{tabular}

Lymph node assessment

Sentinel lymph node 35 (22.9)

ALND 118 (77.1)

Pathologic lymph node status

Negative 91 (59.5)

Positive 62 (40.5)

Extranodal extension

\begin{tabular}{lr} 
Absent & $132(86.3)$ \\
\hline Present & $21(13.7)$
\end{tabular}

Downstage after NAC

No $\quad 52(34.0)$

$\begin{array}{ll}\text { Yes } & 101(66.0)\end{array}$

Surgery type

Breast-conserving surgery 73 (47.7)

Mastectomy $\quad 80(52.3)$

NAC type

\begin{tabular}{ll} 
AC-T & $62(40.5)$ \\
\hline FEC-T & $27(17.6)$ \\
\hline ET & $32(20.9)$ \\
\hline Other & $32(20.9)$
\end{tabular}

Adjuvant chemotherapy

No 107 (69.9)

Yes 46 (30.1)

RT boost

No 52 (34.0)

$\begin{array}{ll}\text { Yes } & 101(66.0)\end{array}$

RT volume

Regional nodal irradiation $124(81.0)$

Whole-breast 29 (19.0)

RT dose, Gy

Median (range)

$50.4(46.0-54.0)$

RT boost dose, Gy

Median (range)

$10.0(4.0-20.0)$

Failures

\begin{tabular}{lr}
\hline In-breast & $5(3.3)$ \\
\hline Chest wall & $11(7.2)$ \\
\hline Regional node & $11(7.2)$
\end{tabular}

Abbreviations: AC-T, doxorubicin/cyclophosphamide/paclitaxel; ALND, axillary lymph node dissection; ET, epirubicin/paclitaxel; FEC-T, fluorouracil/ epirubicin/cyclophosphamide/docetaxel; LVSI, lymphovascular space invasion; $\mathrm{NAC}$, neoadjuvant chemotherapy; RT, radiation therapy. 
A

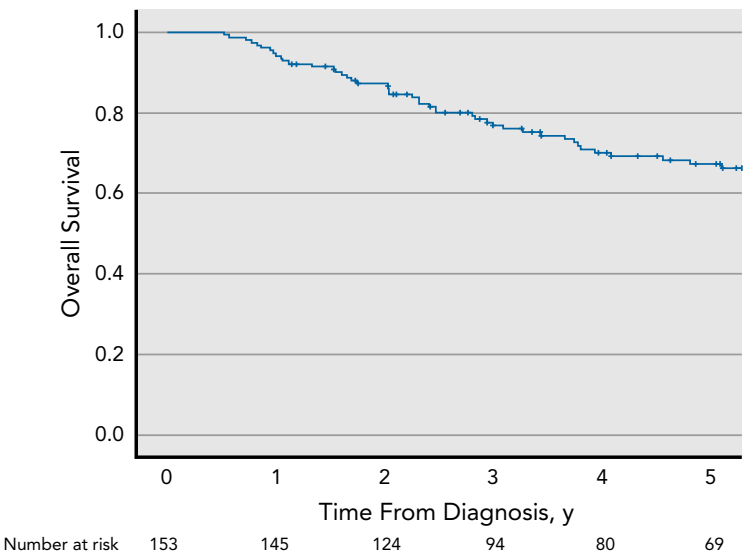

B

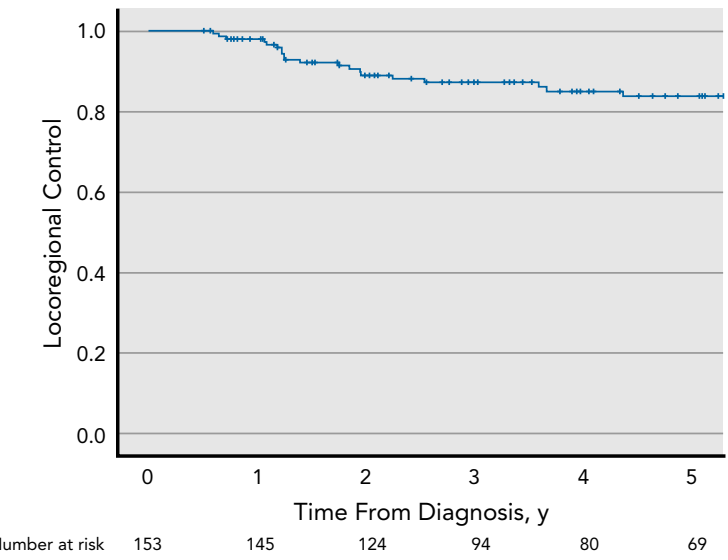

Figure 1. (A) Overall survival and (B) locoregional control in patients with triple-negative breast cancer treated with neoadjuvant chemotherapy.

63 patients who did not have either LVSI or ENE, and of these, only 6 (9.5\%) developed LRR. At 4-year follow-up, patients who had both LVSI and ENE had a lower rate of locoregional control than those with either LVSI or ENE alone, those with neither LVSI nor ENE, and those with pCR (Figure 2D) $(P<.001)$.

\section{Discussion}

This is a large retrospective study evaluating factors predictive of LRR in women with TNBC who do not experience pCR after NAC. We found that in these women, ENE and LVSI are independent predictors of LRR. Patients with both of these risk factors have an especially high rate of LRR. In this cohort, $50 \%$ of patients who had both risk factors developed LRR. If confirmed in other studies, these factors should be considered when recommending adjuvant therapy for patients with TNBC. Therefore, future clinical trials are warranted in this patient population to lower the risk of LRR.

It is well-known that patients who achieve pCR after NAC have favorable outcomes with lower rates of LRR. ${ }^{6,7}$ Analysis of the NSABP B-18 and B-27 trials showed the critical impact of pathologic response to NAC in the breast and axillary lymph nodes on LRR. ${ }^{7}$ In this analysis, patients who did not experience pCR in both breast/ chest wall and lymph nodes were twice as likely to develop LRR as those who did. However, this analysis encompassed all subtypes of breast cancers. In a TNBCspecific cohort of patients receiving NAC, surgery, and RT, Chen et $\mathrm{al}^{12}$ reported that at 2-year follow-up, patients who experienced pCR had a $0 \%$ rate of LRR versus $27.5 \%$ in those who did not. This is similar to our findings, wherein patients who experienced pCR had a $0 \%$ rate of LRR versus $22 \%$ in those who did not. However, these other studies failed to describe features in the residual tumor that are more likely to result in an increased risk of
LRR. Our study went a step further to show that patients with residual disease after NAC who have ENE and/or LVSI have significantly higher rates of LRR than those with residual disease who do not have these pathologic features.

The presence of upfront LVSI is a well-known predictor of LRR in breast cancer, ${ }^{13-16}$ but it is not well evaluated in the post-NAC setting. Post-NAC LVSI was also recently shown by Hamy et $\mathrm{al}^{15}$ to be an independent predictor of disease-free and overall survival in a subgroup of 330 patients with TNBC. Our results are in accordance with this finding, showing that even in patients receiving adjuvant RT, post-NAC LVSI confers a high risk of LRR. Hamy et $\mathrm{al}^{15}$ reported a higher incidence of post-NAC LVSI in patients with low-grade histology, luminal tumors, and a larger number of involved lymph nodes, similar to characteristics of chemoresistant tumors. These findings and ours establish the additional independent risk afforded by LVSI over the simple presence of invasive cancer after NAC. It is theorized that cells that develop the ability to penetrate the vascular endothelium, survive within the intravascular space, and persist through chemotherapy are both biologically aggressive and poised to metastasize regionally and distally, helping to explain the close association between LVSI and LRR. ${ }^{16}$ Therefore, we propose that post-NAC LVSI status should be evaluated in future analyses. If our findings are independently validated in additional prospective analyses, then post-NAC LVSI could be incorporated into future post-NAC prognostic indices to help clinicians make appropriate patient recommendations.

In addition, ENE is known to confer an increased risk of recurrence in those with breast cancer. ${ }^{17,18}$ Even though NAC has been shown to decrease the incidence of ENE found at the time of surgery, ${ }^{19}$ there are few existing data on the prognostic implications of persistent ENE 
A

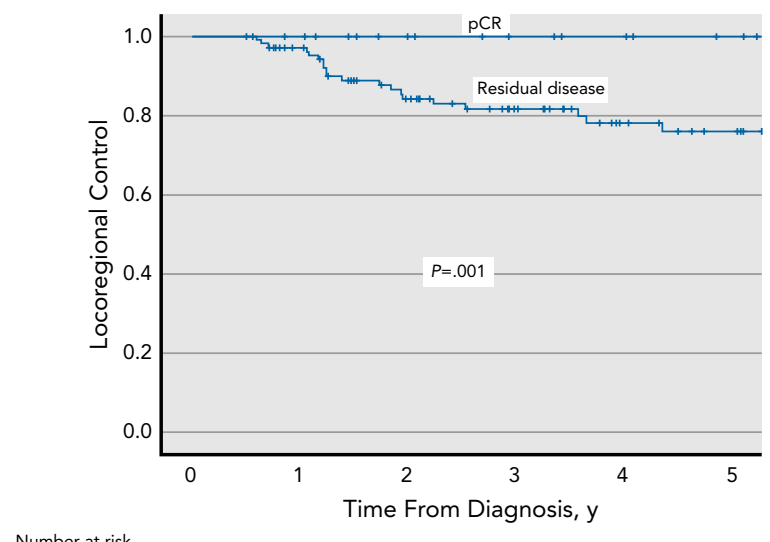

B

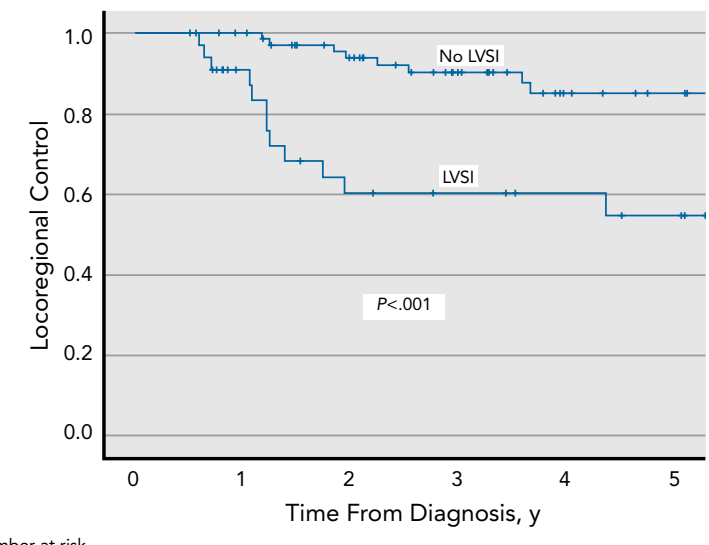

Number at risk

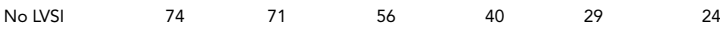
LVSI

D

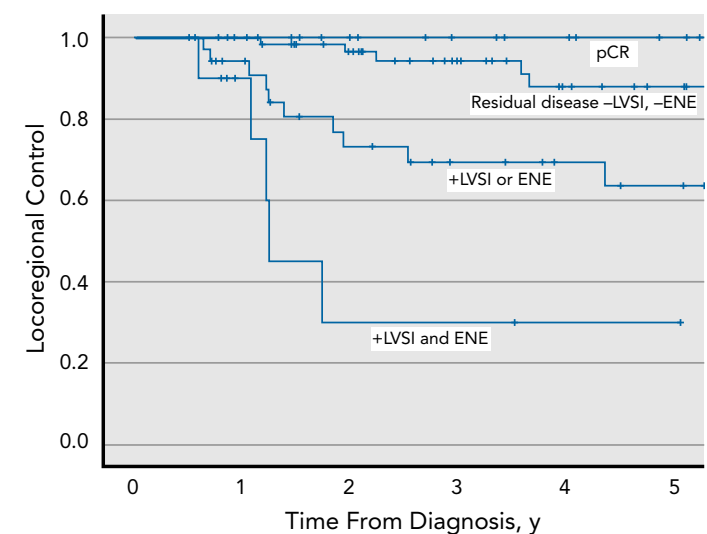

$\begin{array}{lrrrrrr}\text { Number at risk } & & & & & & \\ \text { pCR } & 45 & 44 & 38 & 34 & 32 & 29 \\ \text {-LVSI, ENE } & 63 & 60 & 49 & 36 & 27 & 22 \\ \text { + LVSI or ENE } & 35 & 29 & 20 & 15 & 12 & 10 \\ \text { +LVSI and ENE } & 10 & 6 & 2 & 2 & 1 & 1\end{array}$

Figure 2. Locoregional control in patients with triple-negative breast cancer treated with neoadjuvant chemotherapy (A) among those who experienced pCR versus no PCR; in patients without $P C R$, those with (B) LVSI versus no LVSI and (C) ENE versus no ENE; and (D) comparison of all subgroups.

Abbreviations: ENE, extranodal extension; LVSI, lymphovascular space invasion; $\mathrm{PCR}$, pathologic complete response.

after NAC. This is of particular importance in the TNBC population, because a higher rate of ENE than in estrogen/ progesterone receptor-positive subtypes is reported..$^{20}$ Our study thus reports novel data showing that patients with ENE after NAC have 3 times the increased risk of LRR compared with those with residual disease not extending through the nodal capsule. Multiple potential explanations for this correlation exist. It is possible that occult microscopic tumor persists more commonly in patients with ENE. In addition, tumors with ENE that persist after NAC may simply be more aggressive, radiation-resistant, and likely to recur. As such, we propose that post-NAC ENE also be evaluated and independently verified in prospective studies, similarly to LVSI.
Various strategies of treatment intensification have been evaluated in patients with TNBC to improve outcomes. The addition of carboplatin to standard NAC has produced improved rates of pCR but have an unknown effect on survival. ${ }^{21,22}$ In addition, a recent meta-analysis of 9 randomized clinical trials showed that platinumbased NAC is associated with significantly increased rates of pCR but also with higher rates of hematologic toxicities. ${ }^{23}$ Our analysis raises the interesting question whether the addition of platinum agents to NAC regimens would result in a lower rate of residual LVSI and ENE and corresponding improvements in LRR. This has yet to be evaluated in subset analyses of these studies. A recent trial evaluating the use of PARP inhibitor veliparib in 
Table 2. Factors Predictive of Locoregional Recurrence in Patients With No Pathologic Complete Response

Univariable Analysis

Multivariable Analysis

\begin{tabular}{|c|c|c|c|}
\hline \multirow[b]{2}{*}{ Characteristic } & & \multirow{2}{*}{ HR (95\% } \\
\hline & HR $(95 \% \mathrm{Cl})$ & $P$ Value & \\
\hline \multicolumn{4}{|l|}{ Age, y } \\
\hline$<50$ & Ref & & Ref \\
\hline$\geq 50$ & $2.10(0.87-5.09)$ & .1 & NS \\
\hline \multicolumn{4}{|l|}{ Race } \\
\hline White & Ref & & Ref \\
\hline Black & $1.29(0.55-3.05)$ & .56 & NS \\
\hline \multicolumn{4}{|l|}{ Menopausal status } \\
\hline Postmenopausal & Ref & & Ref \\
\hline Premenopausal & $0.47(0.19-1.13)$ & .09 & NS \\
\hline
\end{tabular}

Clinical T stage

\begin{tabular}{lcccc}
\hline T1-2 & Ref & Ref \\
\hline T3-4 & $1.88(0.76-4.67)$ & .17 & NS
\end{tabular}

Clinical $\mathrm{N}$ stage

\begin{tabular}{lccc}
\hline N0-1 & Ref & Ref \\
\hline N2-3 & $1.90(0.76-4.70)$ & .17 & NS
\end{tabular}

Multifocal

\begin{tabular}{lcccc}
\hline No & Ref & Ref \\
\hline Yes & $2.38(0.91-6.23)$ & .08 & NS
\end{tabular}

Histology

Ductal

Ref

Ref

Other

$0.74(0.17-3.19)$

.69

NS

Grade

\begin{tabular}{lcccc}
\hline I or II & Ref & Ref \\
\hline III & $0.78(0.28-2.12)$ & .62 & NS
\end{tabular}

Pathologic T stage

\begin{tabular}{lcccc}
\hline T0-2 & Ref & Ref \\
\hline T3-4 & $1.39(0.51-3.83)$ & .52 & NS
\end{tabular}

Pathologic N stage

\begin{tabular}{lccc}
\hline N0-1 & Ref & Ref \\
\hline N2-3 & $1.95(0.75-5.12)$ & .17 & NS
\end{tabular}

Margins

\begin{tabular}{lccc} 
Negative & Ref & Ref \\
\hline Positive & $0.91(0.21-3.90)$ & .9 & NS
\end{tabular}

Lymph node assessment

Sentinel lymph node

Ref

$1.08(0.36-3.23)$

.89

Ref

ALND

Pathologic lymph status

\begin{tabular}{lccc}
\hline Negative & Ref & Ref \\
\hline Positive & $2.48(0.96-6.40)$ & .06 & NS
\end{tabular}

LVSI

Absent

Ref

Present

$4.18(1.76-9.96)$

.001

Ref

$3.92(1.64-9.38)$

.002

(continued on next page)

Abbreviations: AC-T, doxorubicin/cyclophosphamide/paclitaxel; ALND, axillary lymph node dissection; ET, epirubicin/paclitaxel; FEC-T, fluorouracil/epirubicin/ cyclophosphamide/docetaxel; HR, hazard ratio; LVSI, lymphovascular space invasion; NAC, neoadjuvant chemotherapy; NS, not significant; RT, radiation therapy. 


\begin{tabular}{|c|c|c|c|c|}
\hline \multirow[b]{2}{*}{ Characteristic } & \multicolumn{2}{|c|}{ Univariable Analysis } & \multicolumn{2}{|c|}{ Multivariable Analysis } \\
\hline & HR $(95 \% \mathrm{Cl})$ & $P$ Value & HR $(95 \% \mathrm{Cl})$ & $P$ Value \\
\hline \multicolumn{5}{|l|}{ Extranodal extension } \\
\hline Absent & Ref & & Ref & \\
\hline Present & $3.64(1.50-8.85)$ & .004 & $3.32(1.35-8.15)$ & .009 \\
\hline \multicolumn{5}{|l|}{ Downstage after NAC } \\
\hline No & Ref & & Ref & \\
\hline Yes & $0.49(0.20-1.18)$ & .11 & NS & \\
\hline \multicolumn{5}{|l|}{ Surgery type } \\
\hline Breast-conserving surgery & Ref & & Ref & \\
\hline Mastectomy & $1.18(0.49-2.85)$ & .71 & NS & \\
\hline \multicolumn{5}{|l|}{ NAC type } \\
\hline AC-T & Ref & & Ref & \\
\hline FEC-T & $0.70(0.19-2.56)$ & .59 & NS & \\
\hline ET & $0.63(0.20-2.04)$ & .45 & NS & \\
\hline Other & $0.67(0.21-2.14)$ & .5 & NS & \\
\hline \multicolumn{5}{|l|}{ Adjuvant chemotherapy } \\
\hline No & Ref & & Ref & \\
\hline Yes & $0.54(0.21-1.38)$ & .2 & NS & \\
\hline \multicolumn{5}{|l|}{ RT boost } \\
\hline No & Ref & & Ref & \\
\hline Yes & $1.21(0.49-3.00)$ & .68 & NS & \\
\hline \multicolumn{5}{|l|}{ RT volume } \\
\hline Regional nodal irradiation & Ref & & Ref & \\
\hline Whole-breast & $0.74(0.22-2.52)$ & .63 & NS & \\
\hline
\end{tabular}

Abbreviations: AC-T, doxorubicin/cyclophosphamide/paclitaxel; ALND, axillary lymph node dissection; ET, epirubicin/paclitaxel; FEC-T, fluorouracil/epirubicin/ cyclophosphamide/docetaxel; HR, hazard ratio; LVSI, lymphovascular space invasion; NAC, neoadjuvant chemotherapy; NS, not significant; RT, radiation therapy.

combination with carboplatin and paclitaxel in the neoadjuvant setting showed that pCR rates were driven mainly by carboplatin and not by the PARP inhibitor. ${ }^{24}$ Trials studying immunotherapeutic agents in the neoadjuvant setting are also underway.

The question whether the addition of platinum in the adjuvant setting improves outcomes in patients with residual disease after NAC has yet to be evaluated in a prospective trial. Recently published data from the CREATE- $X$ trial showed a survival benefit with adjuvant capecitabine in HER2-negative patients with residual disease after NAC. ${ }^{25}$ Subanalysis of this trial suggests that this benefit was driven primarily by the benefit of capecitabine in the TNBC cohort. However, the practice of administering capecitabine in the adjuvant setting for TNBC remains controversial, as evidenced by the recommendation in the NCCN Clinical Practice Guidelines in Oncology for Breast Cancer to "consider adjuvant capecitabine in patients with TNBC" who received standard neoadjuvant treatment. ${ }^{26}$ Patients in our study were treated before publication of the CREATE-X results, and a minority received adjuvant chemotherapy; however, none received capecitabine in the adjuvant setting. In our study, the presence of LVSI and ENE at the time of surgery was associated with absolute increases in LRR rates of $25 \%$ and $32 \%$, respectively. Based on these data, we propose that the presence of LVSI and/or ENE in residual disease be further studied. If these findings are prospectively validated, then LVSI and ENE can be considered when discussing recommendations with patients regarding adjuvant chemotherapy such as capecitabine.

Use of sentinel lymph node biopsy with NAC is controversial. ${ }^{27}$ The ACOSOG Z1071 trial showed a slightly higher false-negative rate of sentinel lymph nodes after NAC; however, it was $<10 \%$ if $\geq 2$ sentinel nodes were removed. ${ }^{28}$ In this analysis, $22.9 \%$ of patients underwent sentinel lymph node biopsy. Notably, LRR in our study occurred despite all patients receiving adjuvant 
RT. Relative radioresistance of the TNBC subtype compared with other breast cancer subtypes has previously been reported. ${ }^{5}$ Therefore, the addition of concurrent radiosensitizing agents may be the preferred method of enhancing locoregional control in patients with residual disease after NAC, particularly when LVSI or ENE is present. Concurrent radiosensitizing chemotherapy and RT have demonstrated favorable local disease response in locally advanced breast cancer in the upfront setting and in patients whose disease is resistant to firstline chemotherapy. ${ }^{29-31}$ Likewise, hyperthermia is a well-known radiosensitizer, and its combination with RT has demonstrated improved local control compared with RT alone in randomized studies, ${ }^{32,33}$ with acceptable toxicity profiles. ${ }^{34}$ Therefore, treatment with concurrent radiosensitizing chemotherapy or hyperthermia should also be considered in future prospective trials for patients with TNBC who have residual LVSI and/or ENE after NAC.

Given the retrospective nature of this series, our study has several inherent limitations. Our data represent patients from a single institution, and treatment regimens at other institutions may differ and result in different outcomes. Therefore, our results should be interpreted with caution, and future larger prospective trials should be considered to validate our findings. In addition, our analysis of only 153 patients may limit the power to detect other meaningful predictors of LRR.

\section{Conclusions}

Our study results show that ENE and LVSI in residual breast cancer after NAC predict increased risk of LRR in patients with TNBC. On prospective validation, these factors can be used by clinicians to counsel their patients and make appropriate recommendations for adjuvant treatment or surveillance. For these patients, additional adjuvant chemotherapy, and the addition of radiosensitizing agents concurrently with RT, may be warranted to lower the risk of LRR in future prospective clinical trials.

\section{Acknowledgments}

The authors wish to acknowledge the patients in this study, Siteman Cancer Center, and the Department of Radiation Oncology for their support of this study.

Submitted April 24, 2018; accepted for publication November 5, 2018.

Author contributions: Study concept and design: Gabani, Merfeld, Zoberi. Data acquisition: Gabani, Merfeld, Weiner, Mullen. Data analysis and interpretation: Gabani, Merfeld, Srivastava, Weiner, Zoberi. Manuscript preparation: Gabani, Merfeld. Critical revision: Ochoa, Thomas, Margenthaler, Cyr, Peterson, Naughton, Ma, Zoberi.

Disclosures: The authors have not received any financial consideration from any person or organization to support the preparation, analysis, results, or discussion of this article.

Funding: The Siteman Cancer Center is supported by NCl Cancer Center Support Grant P30 CA91842. Funds to conduct this study were supplied by the Department of Radiation Oncology.

Correspondence: Imran Zoberi, MD, Department of Radiation Oncology, Washington University School of Medicine, 4921 Parkview Place, Saint Louis, MO 63110. Email: izoberi@wustl.edu

\section{References}

1. Foulkes WD, Smith IE, Reis-Filho JS. Triple-negative breast cancer. N Engl J Med 2010;363:1938-1948.

2. Liedtke C, Mazouni C, Hess KR, et al. Response to neoadjuvant therapy and long-term survival in patients with triple-negative breast cancer. J Clin Oncol 2008;26:1275-1281.

3. McGuire A, Lowery AJ, Kell MR, et al. Locoregional recurrence following breast cancer surgery in the trastuzumab era: a systematic review by subtype. Ann Surg Oncol 2017;24:3124-3132.

4. Caudle AS, Yu TK, Tucker SL, et al. Local-regional control according to surrogate markers of breast cancer subtypes and response to neoadjuvant chemotherapy in breast cancer patients undergoing breast conserving therapy. Breast Cancer Res 2012;14:R83.

5. Kyndi M, Sørensen FB, Knudsen H, et al. Estrogen receptor, progesterone receptor, HER-2, and response to postmastectomy radiotherapy in highrisk breast cancer: the Danish Breast Cancer Cooperative Group. J Clin Oncol 2008;26:1419-1426.

6. Chaudhary LN, Wilkinson $\mathrm{KH}$, Kong A. Triple-negative breast cancer: who should receive neoadjuvant chemotherapy? Surg Oncol Clin N Am 2018; 27:141-153.

7. von Minckwitz G, Untch M, Blohmer JU, et al. Definition and impact of pathologic complete response on prognosis after neoadjuvant chemotherapy in various intrinsic breast cancer subtypes. J Clin Oncol 2012;30: 1796-1804.

8. Mamounas EP, Anderson SJ, Dignam JJ, et al. Predictors of locoregional recurrence after neoadjuvant chemotherapy: results from combined analysis of National Surgical Adjuvant Breast and Bowel Project B-18 and B-27. J Clin Oncol 2012;30:3960-3966.

9. Xiangying $\mathrm{M}$, Shikai $\mathrm{W}$, Zefei J, et al. Progestin as an alternative treatment option for multi-treated recurrent triple-negative breast cancer. Swiss Med Wkly 2013;143:w13765
10. Braunstein $L Z$, Niemierko A, Shenouda $M N$, et al. Outcome following local-regional recurrence in women with early-stage breast cancer: impact of biologic subtype. Breast J 2015;21:161-167.

11. Aft R, Naughton M, Trinkaus K, et al. Effect of zoledronic acid on disseminated tumour cells in women with locally advanced breast cancer: an open label, randomised, phase 2 trial. Lancet Oncol 2010; 11:421-428.

12. Chen VE, Gillespie EF, Zakeri K, et al. Pathologic response after neoadjuvant chemotherapy predicts locoregional control in patients with triple negative breast cancer. Adv Radiat Oncol 2017;2: 105-109.

13. Abdulkarim BS, Cuartero J, Hanson J, et al. Increased risk of locoregional recurrence for women with T1-2N0 triple-negative breast cancer treated with modified radical mastectomy without adjuvant radiation therapy compared with breast-conserving therapy. J Clin Oncol 2011;29: 2852-2858.

14. Chen X, Yu X, Chen J, et al. Analysis in early stage triple-negative breast cancer treated with mastectomy without adjuvant radiotherapy: patterns of failure and prognostic factors. Cancer 2013;119:2366-2374.

15. Hamy AS, Lam GT, Laas E, et al. Lymphovascular invasion after neoadjuvant chemotherapy is strongly associated with poor prognosis in breast carcinoma. Breast Cancer Res Treat 2018;169:295-304.

16. Aleskandarany MA, Sonbul SN, Mukherjee A, et al. Molecular mechanisms underlying lymphovascular invasion in invasive breast cancer. Pathobiology 2015;82:113-123.

17. Choi AH, Surrusco M, Rodriguez S, et al. Extranodal extension on sentinel lymph node dissection: why should we treat it differently? Am Surg 2014; 80:932-935.

18. Nottegar A, Veronese N, Senthil M, et al. Extra-nodal extension of sentinel lymph node metastasis is a marker of poor prognosis in breast cancer 
patients: a systematic review and an exploratory meta-analysis. Eur J Surg Oncol 2016;42:919-925.

19. Al-Hilli Z, Hieken TJ, Hoskin TL, et al. Impact of neoadjuvant chemotherapy on pathologic axillary nodal status in HER-2 positive patients presenting with clinically node-negative disease. J Surg Oncol 2015;112: 453-457.

20. Ibrahim E, Al-Gahmi AM, Zeenelin AA, et al. Basal vs. luminal A breast cancer subtypes: a matched case-control study using estrogen receptor, progesterone receptor, and HER-2 as surrogate markers. Med Oncol 2009;26:372-378.

21. Sikov WM, Berry DA, Perou CM, et al. Impact of the addition of carboplatin and/or bevacizumab to neoadjuvant once-per-week paclitaxel followed by dose-dense doxorubicin and cyclophosphamide on pathologic complete response rates in stage II to III triple-negative breast cancer: CALGB 40603 (Alliance). J Clin Oncol 2015;33:13-21.

22. von Minckwitz G, Schneeweiss A, Loibl S, et al. Neoadjuvant carboplatin in patients with triple-negative and HER2-positive early breast cancer (GeparSixto; GBG 66): a randomised phase 2 trial. Lancet Oncol 2014;15: 747-756.

23. Poggio F, Bruzzone M, Ceppi M, et al. Platinum-based neoadjuvant chemotherapy in triple-negative breast cancer: a systematic review and meta-analysis. Ann Oncol 2018;29:1497-1508.

24. Loibl S, O'Shaughnessy J, Untch M, et al. Addition of the PARP inhibitor veliparib plus carboplatin or carboplatin alone to standard neoadjuvant chemotherapy in triple-negative breast cancer (BrighTNess): a randomised, phase 3 trial. Lancet Oncol 2018;19:497-509.

25. Masuda N, Lee SJ, Ohtani S, et al. Adjuvant capecitabine for breast cancer after preoperative chemotherapy. N Engl J Med 2017;376:2147-2159.

26. Gradishar WJ, Anderson BO, Abraham J, et al. NCCN Clinical Practice Guidelines in Oncology: Breast Cancer. Version 4.2018. Accessed April
15,2018 . To view the most recent version of these guidelines, visit NCCN.org.

27. Yan M, Abdi MA, Falkson C. Axillary management in breast cancer patients: a comprehensive review of the key trials. Clin Breast Cancer 2018; 18:e1251-1259.

28. Boughey JC, Suman VJ, Mittendorf EA, et al. Sentinel lymph node surgery after neoadjuvant chemotherapy in patients with node-positive breast cancer: the ACOSOG Z1071 (Alliance) clinical trial. JAMA 2013;310: 1455-1461.

29. Brackstone $M$, Palma $D$, Tuck $A B$, et al. Concurrent neoadjuvant chemotherapy and radiation therapy in locally advanced breast cancer. Int $\mathrm{J}$ Radiat Oncol Biol Phys 2017;99:769-776.

30. Woodward WA, Fang P, Arriaga L, et al. A phase 2 study of preoperative capecitabine and concomitant radiation in women with advanced breast cancer. Int J Radiat Oncol Biol Phys 2017;99:777-783.

31. Gaui MF, Amorim G, Arcuri RA, et al. A phase II study of second-line neoadjuvant chemotherapy with capecitabine and radiation therapy for anthracycline-resistant locally advanced breast cancer. Am J Clin Oncol 2007;30:78-81.

32. Vernon CC, Hand JW, Field SB, et al. Radiotherapy with or without hyperthermia in the treatment of superficial localized breast cancer: results from five randomized controlled trials. Int J Radiat Oncol Biol Phys 1996; 35:731-744.

33. Jones EL, Oleson JR, Prosnitz LR, et al. Randomized trial of hyperthermia and radiation for superficial tumors. J Clin Oncol 2005;23:3079-3085.

34. Varma S, Myerson R, Moros E, et al. Simultaneous radiotherapy and superficial hyperthermia for high-risk breast carcinoma: a randomised comparison of treatment sequelae in heated versus non-heated sectors of the chest wall hyperthermia. Int J Hyperthermia 2012;28:583-590. 